\title{
Potential new drug for leukaemia
}

\author{
Eileen Russell
}

Department of Biochemistry, UCC

It's 1963 and the parents of Edward Burke try to make their child as comfortable as possible. It is a difficult task given that the 4 year olds pale body is covered in melon sized bruises. His bleeding gums briefly distract them from his persistent coughing and fever. The fair-haired boys meek voice complains of pain all over his swollen belly. This is leukaemia, for which there was no cure.

\section{What is leukaemia?}

Leukaemia is a cancer of the blood. Cancer is a group of diseases characterized by unregulated cell growth. There are over 200 different types of cancer, each classified by the type of cell that is affected. Blood is composed of red cells, white cells, platelets and plasma. These components are marked in figure 1. White blood cells play a vital role in fighting infection. In leukaemia, there is an unregulated increase in abnormal white blood cells. This explains where the term 'leukaemia' originated as it comes from the Greek words "leukos" and "heima," also meaning "white blood". These abnormal white blood cells, or leukaemic cells, grow rapidly and crowd out the normal cells that the body requires to function properly. In addition, leukaemic cells can move from the blood to other parts of the body. This movement, known as metastasis, allows the cancer to spread.

Factors that may affect the development of leukaemia are unclear but are thought to include family history and exposure to certain chemicals and radiation. Symptoms of the disease include tiredness, bruising, regular infections, fever and pain.

\section{Leukaemia as a model for other cancers}

Although leukaemia develops far less frequently than many other cancers, research in this disease has been some of the most productive of all cancer research. Virtually all of the major advances in cancer were first made in blood-related cancers, and subsequently transferred to other diseases. For example, the first chemotherapy was developed in leukaemia patients. The main reason that leukemia has been so productive for scientific discovery is that the blood provides great flexibility for researchers. Blood cells can easily be obtained from patients, and continue to be studied once outside the body. Cancerous blood cells can be drawn regularly from a patient receiving treatment with very little pain to the patient. 


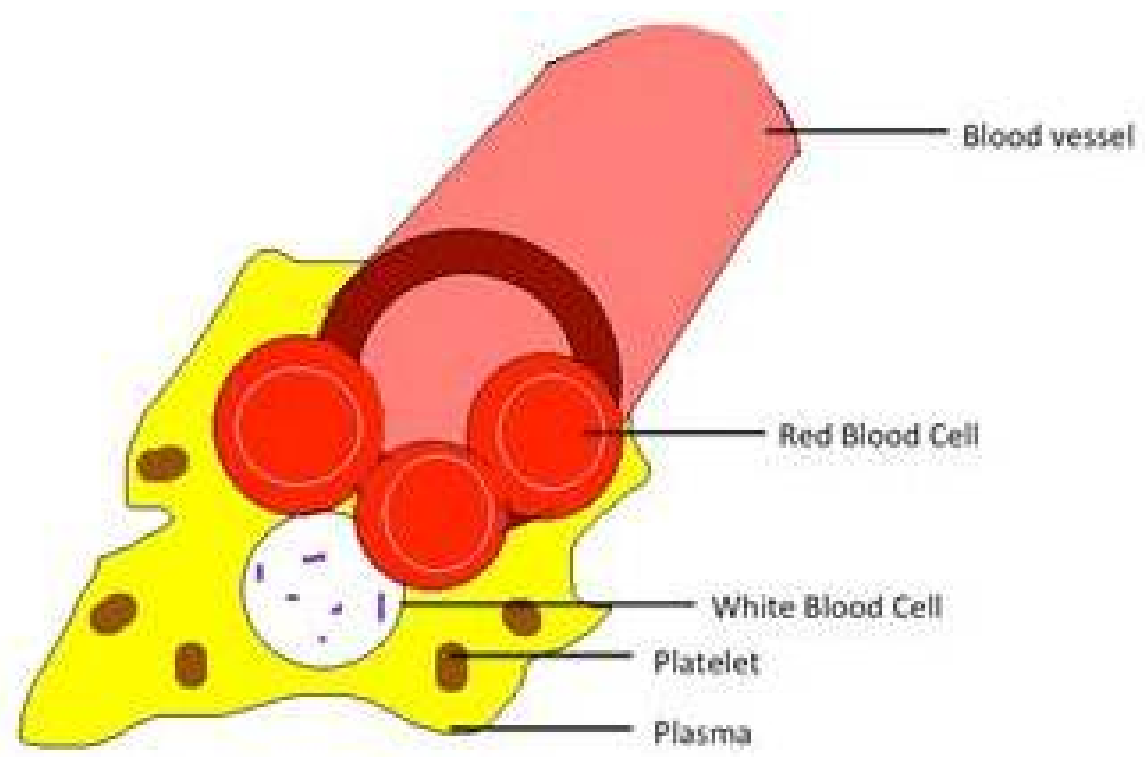

Figure 1: Components of the blood. Image: Eileen Russell.

\section{Treatment of Leukaemia}

Chemotherapy is the main treatment approach for leukaemia. The aim of chemotherapy is to kill the cancer cells while doing the least possible damage to normal cells. Figure 2 shows a cancer cell undergoing cell death. The image was captured by my supervisor, Professor Tom Cotter. A combination of chemotherapeutic drugs is often given to a patient. There are a number of chemotherapy drugs available, however, their effects are not always long lasting. The effects are usually so short lived due to a phenomenon called 'resistance'. Resistance occurs when cancer cells resist the effects of chemotherapy, all it takes is a few drug-defiant cells to thrive. Undoubtedly, leukaemia treatment has come a long way since it was first observed in 1845, but there is clear need to develop better drugs for this disease.
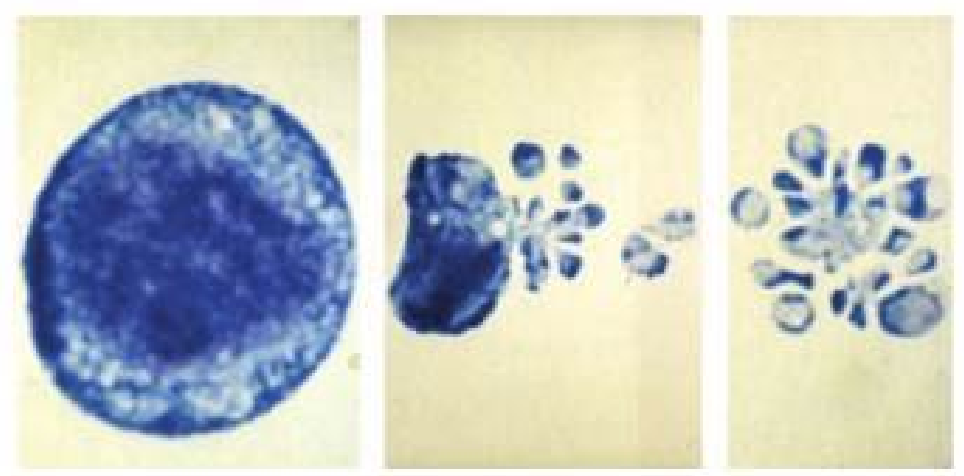

Figure 2: A cancer cell undergoing cell death. Image: Prof. Tom Cotter. 
Untreated cells

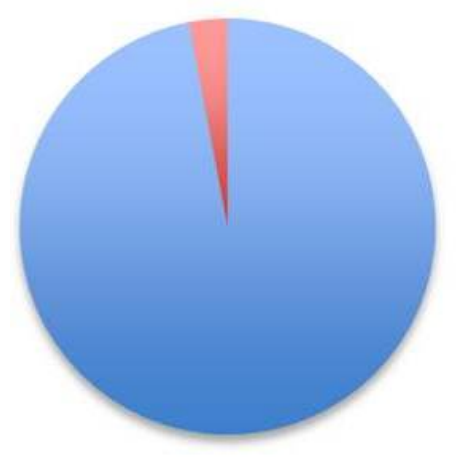

\section{Cells treated with isoellipticine}

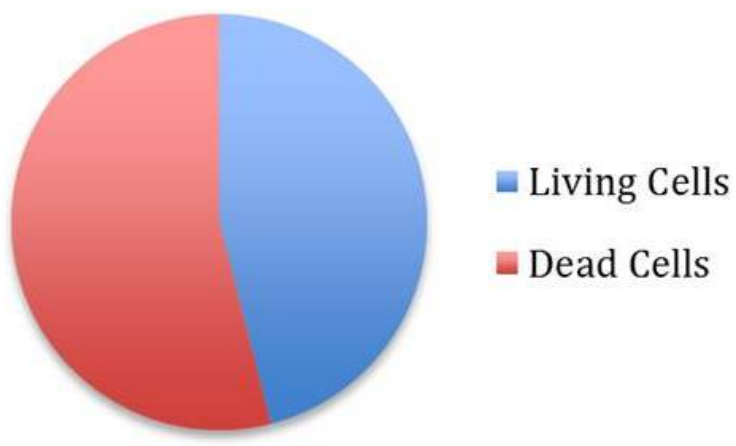

Figure 3: DIfference in health of treated and untreated cells. Image: Eileen Russell.

\section{My Research}

My project aims to improve ellipticine, an existing drug whose anti-cancer properties are already well known and characterized.

Ellipticine was first isolated from the leaf of Ochrosia elliptica Labill by Goodwin in 1959. Ellipticine was found to display strong anticancer activity. Unfortunately, ellipticine's use is limited as only a small fraction of the drug enters the blood. However, making a small structural change to ellipticine rectifies this problem. The modified ellipticine is called isoellipticine. My project is a partnership between our cancer biology laboratory and a chemistry laboratory in UCC. The chemistry group perform experiments on the drug to alter it before passing it over to us. We examine how good the altered drug is at killing leukaemia cells. So far, results show that the changes we have made to the structure of ellipticine have made it more effective at killing leukaemia cells. This is demonstrated in figure 3 which shows that cells treated with isoellipticine show 20 times more of a response than those not treated. Excitingly, preliminary data also suggests that isoellipticine has beneficial effects when used with an existing chemotherapeutic drug. Research is also being carried out to discover the best dose and how often the drug should be administered. All of the experiments in my project work with cells derived from leukaemia patients. These cells are now grown outside of the body in controlled conditions. This technique is known as cell culture. Cell culture involves the growth of cells in an environment composed of nutrient solutions and ideal conditions of temperature, humidity and gaseous atmosphere. This system allows a researcher to measure the response of the cell's alterations in prospective drugs. The advantage of these kind of experiments is that they permit simplification of a system. Armed with the knowledge gained from cell culture experiments, scientists can move forward to other experiments with isoellipticine. 


\section{What next?}

The drug discovery process is lengthy. If a drug is found to be effective in cells outside of the body, it must then be used in experiments in cells that are in a living body. Following successful completion of this process the drug will move into trials in patients. Regulatory bodies must then review the drug's performance. Even after the approval of a drug, postmarketing surveillance takes place. While most drugs don't make it to the end of the process, research on these drugs can still be constructive. For example, if a drug fails to work, it may still tell us something new about the disease and work can begin on designing a drug overcoming this. The process can be tedious but patient safety is priority. It is laboratory experiments such as those carried out in my project, which lay the foundation in the drug discovery process.

\section{Summary}

Since Edward Burke's death in 1964, survival rates for leukaemia have quadrupled. A diagnosis of leukaemia is life changing but is no longer a death sentence thanks to advances in research. There is still an urgent need for a drug to combat leukaemia to further increase the survival of patients. It is hoped that data obtained from our research can simultaneously contribute to the knowledge about the disease and lead to the development of a new drug. Such a drug would improve the survival chances, and quality of life of people with the disease. This may be beneficial to sufferers of other types of cancer as many leukaemia phenomenona are applicable across to many categories of the disease.

Thanks to my supervisor Professor Tom Cotter, Dr. Florence McCarthy, my colleagues and my funding body, PRTLI. 